\title{
Caracterización del conjugado no covalente de grafeno y ácido fólico mediante espectroscopia Raman y métodos computacionales
}

\author{
John J. Castillo ${ }^{1,3, *}$, Angie E. Orduz ${ }^{1}$, Ciro E. Rozo ${ }^{2}$ \\ ${ }^{1}$ Grupo de Investigación en Bioquímica y Microbiología, GIBIM, Universidad Industrial de Santander, Bucaramanga, Colombia \\ ${ }^{2}$ Grupo de Investigaciones Ambientales para el Desarrollo Sostenible, Facultad de Química Ambiental, \\ Universidad Santo Tomas, Floridablanca, Colombia \\ ${ }^{3}$ Department of Micro and Nanotechnology, Technical University of Denmark, Lyngby, Denmark
}

\begin{abstract}
Resumen
La preparación de compuestos híbridos de biomoléculas y estructuras de tamaño nanométrico es de especial interés para el desarrollo de aplicaciones en el campo de los biosensores y la entrega de fármacos, entre otros. En este estudio se llevó a cabo la preparación de un conjugado formado por grafeno y ácido fólico cuya caracterización se hizo mediante espectroscopia Raman y el método computacional de la teoría del funcional de la densidad (DFT). El conjugado no covalente se obtuvo mediante un método rápido y sencillo de tipo "one pot". La espectroscopia Raman permitió evidenciar la funcionalización del grafeno con el ácido fólico mediante el análisis de las relaciones en la intensidad de las señales Raman correspondiente a las bandas G y D. El método DFT mostró que la unión de grafeno y ácido fólico ocurre por la acción de fuerzas de enlace de tipo no covalente, como las interacciones de tipo $\pi-\pi$, y de puentes de hidrógeno. La obtención de este tipo de conjugados permitiría explorar su aplicación en el diseño de biosensores que detecten células cancerígenas con sobreexpresión de receptores de folato, con el fin de facilitar un diagnóstico eficaz y oportuno del cáncer. (C) 2018. Acad. Colomb. Cienc. Ex. Fis. Nat.
\end{abstract}

Palabras claves: Grafeno; Espectroscopia Raman; Ácido fólico; Métodos computacionales.

Characterization of the non-covalent conjugate graphene-folic acid using Raman spectroscopy and computational methods

\begin{abstract}
Hybrid compounds formed by biomolecules and nanometric structures are attracting interest for the development of applications in the fields of biosensors and drug delivery. In this study we prepared a grapheme-folic acid conjugate which we characterized by Raman spectroscopy and the density functional theory (DFT) computational method. The non-covalent conjugate was performed through a simple and fast one-pot method. The analysis of ratio intensity of $\mathrm{D}$ and $\mathrm{G}$ bands in the Raman spectroscopy evidenced graphene functionalization. The DFT method showed that the bond between graphene and folic acid occurs by non-covalent $\pi-\pi$ and hydrogen bond interactions. Graphene and folic acid conjugates will allow the design of biosensors for the detection of cancer cells overexpressing folate receptors using analytic devices for a faster, more effective and timely diagnosis of cancer. (C) 2018. Acad. Colomb. Cienc. Ex. Fis. Nat.
\end{abstract}

Key words: Graphene; Raman spectroscopy; Folic acid; Computational methods.

\section{Introducción}

En los últimos años ha habido un gran interés por la fabricación de nanocompuestos conjugados con biomoléculas para el diagnóstico y tratamiento de diversos tipos de enfermedades. La atención de la comunidad científica se centra en aquellos nanomateriales con propiedades fisicoquímicas y biológicas que los hacen aptos para las aplicaciones biomédicas. El éxito de la nanoestructuración mediante el uso de nanomateriales, como aquellos a base de carbono, se basa en las propiedades únicas del nanomaterial, como una óptima relación entre área y superficie, la elevada energía superficial y la relación ente superficie y volumen, la estabilidad térmica, la fácil funcionalización y, lo más importante, la poca toxicidad a la hora de realizar experimentos que involucren su interacción con células y diferentes tipos de microorganismos.

El grafeno es una forma alotrópica de carbono compuesta exclusivamente por átomos de carbono con un patrón

\footnotetext{
*Correspondencia:
}

John J. Castillo, jcasleon@uis.edu.co

Recibido: 22 de septiembre de 2017

Aceptado: 06 de marzo de 2018

Editor: Édgar Páez Mozo 
regular hexagonal, similar al del grafito, pero en una hoja de un átomo de espesor (Allen, et al., 2010). Entre los métodos de obtención del grafeno se destacan la exfoliación con cinta adhesiva del grafito, la exfoliación con solvente, la deposición por vapor químico, la descarga de arco eléctrico y la limpieza por ultrasonido (Allen, et al., 2010). Es un nanomaterial muy resistente, flexible y elástico y, además, posee excelentes propiedades de conducción térmica y eléctrica. En medicina se ha utilizado principalmente como óxido de grafeno para el diseño de dispositivos de transporte de fármacos hacia células cancerígenas (Hu, et al., 2013).

Uno de los mayores desafíos con las nanoestructuras es la posibilidad de funcionalizarlas con biomoléculas para así ampliar el espectro de aplicaciones. Precisamente gracias a la estructura del grafeno ha sido posible utilizar su forma oxidada y reducida en la conjugación con proteínas, enzimas, ADN, virus, etc. (Balapanuru, et al., 2010). La presencia de diferentes grupos funcionales en su estructura permite crear uniones covalentes con biomoléculas. Sin embargo, en ocasiones la creación de funcionalidades en el grafeno requiere métodos químicos agresivos que provocan daños en su estructura electrónica. Por otro lado, existe también la funcionalización no covalente, la cual se basa en interacciones del tipo van der Waals, las hidrofóbicas y las de tipo $\pi-\pi$. Una de las ventajas de esta modalidad de funcionalización es que la estructura aromática del grafeno se preserva, sin modificar su estructura electrónica (Bose, et al., 2011). Entre las metodologías más utilizadas para la funcionalización no covalente están aquellas que hacen uso de surfactantes y algunas biomoléculas como el ADN, los carbohidratos, las proteínas, etc. (Kamińska, et al., 2011). Algunas moléculas de la familia de los pirenos se han utilizado exitosamente para la funcionalización no covalente de nanotubos de carbono (NTC) en aplicaciones de biosensores, uniendo la parte planar o aromática de la molécula de pireno al NTC mediante interacciones de apilamiento (Choi, et al., 2010).

El ácido fólico es una de las biomoléculas más utilizadas en aplicaciones biomédicas; además, es una molécula de vital importancia en la síntesis del ADN y un suplemento vitamínico que ayuda a las mujeres en embarazo a prevenir defectos congénitos en los recién nacidos (Castillo, et al., 2015). Se ha utilizado como blanco estratégico en la detección, diagnóstico y tratamiento en enfermedades como el cáncer, ya que en los últimos años se ha establecido que reconoce ciertas células cancerígenas que presentan receptores de folatos, interacción que se caracteriza por una constante de disociación $(\mathrm{Kd})$ de $1 \mathrm{nM}$. La interacción entre el ácido fólico y los receptores de folatos se ha aprovechado para preparar sistemas de entrega selectiva de fármacos en células cancerígenas utilizando grafeno o nanotubos de carbono (Castillo, et al., 2013). Otra estrategia utiliza nanocompuestos funcionalizados con este ácido para preparar sistemas de detección rápida de células cancerígenas o parásitos involucrados en diferentes tipos de enfermedades, como es el caso de la leishmaniasis (Castillo, et al., 2012)
Los conjugados formados por nanocompuestos y biomoléculas pueden caracterizarse para así evidenciar la unión del enlace formado. Ello puede hacerse con técnicas tradicionales, como la resonancia magnética nuclear (RMN), la espectroscopia ultravioleta-visible (UV-Vis) y la infrarroja (IR), pero debido a que se trata de nanoestructuras, su caracterización exige el uso de técnicas sofisticadas como el análisis termogravimétrico (ATG), la espectroscopia de difusión ordenada (DOSY 2D) por RMN en dos dimensiones (2D), la espectroscopia Raman, la microscopía electrónica de barrido (SEM), la microscopía electrónica de transmisión (TEM) y la microscopía de fuerza atómica (AFM) (Castillo, et al., 2012). La espectroscopia Raman (ER) es una técnica basada en el fenómeno de dispersión inelástica de luz monocromática en el rango visible, la cual permite el análisis de los modos de vibración y rotación de baja frecuencia de diferentes tipos de moléculas. Algunos estudios han hecho uso de la ER para evidenciar la funcionalización de nanocompuestos con diferentes tipos de biomoléculas. Por ejemplo, Castillo, et al. (2013) utilizaron la relación de intensidades de los picos Raman D y G de los NTC para monitorizar su funcionalización con ácido fólico. En otros trabajos esta misma relación se ha utilizado para evaluar la unión de diferentes proteínas a nanoestructuras formadas por átomos de carbono (Casiraghi, et al., 2011). Asimismo, cabe mencionar la importancia que han cobrado en los últimos años los métodos computacionales para simular las interacciones entre nanoestructuras a base de carbono y diferentes tipos de biomoléculas. Por ejemplo, en el primero de estos estudios se simuló un conjugado formado por NTC y ácido fólico utilizando el método DFT y el funcional de correlación B3LYP con la función base [6-31G[d], y se concluyó que la unión ocurría a través de puentes de hidrógeno (Castillo, et al., 2013).

A pesar de que se han publicado varios estudios sobre la caracterización de nanoestructuras de carbono funcionalizados con ácido fólico, no se encontraron publicaciones que reportaran la caracterización del conjudado de grafeno y ácido fólico mediante ER y métodos computacionales. En este trabajo se combinaron las excelentes propiedades fisicoquímicas y biológicas del conjugado de grafeno y ácido fólico formado mediante una interacción de tipo no covalente. En su caracterización se emplearon métodos espectroscópicos y computacionales que evidenciaron la unión del ácido fólico al grafeno. La obtención y posterior caracterización de este conjugado abren nuevas posibilidades para la fabricación de potenciales sistemas de detección y diagnóstico temprano y oportuno de enfermedades como el cáncer.

\section{Materiales y métodos}

Preparación del conjugado de grafeno y ácido fólico. En un recipiente de vidrio de $50 \mathrm{ml}$ se colocaron cinco miligramos de grafeno y se adicionaron a una solución ligeramente básica $(\mathrm{pH}$ 9) de ácido fólico $(5 \mathrm{ml})$. Para la preparación 
de la solución de ácido fólico se pesaron 6,5 mg del ácido y se adicionaron $10 \mu \mathrm{l}$ de hidróxido de sodio $(\mathrm{NaOH}) 1 \mathrm{M}$ para solubilizarlo, y se añadió agua destilada hasta obtener $25 \mathrm{ml}$ de volumen. La mezcla de grafeno y ácido fólico se agitó durante 5 minutos y posteriormente fue sonicada durante 20 minutos. Durante la sonicación la solución se introdujo en un baño termostático $\left(15^{\circ} \mathrm{C}\right)$ para evitar el sobrecalentamiento generado por el sonicador y evitar que se afectara la estructura electrónica del grafeno. Luego de la sonicación, la solución se centrifugó a $2.800 \mathrm{rpm}$ durante 20 minutos, y el sobrenadante se separó del sólido. Por último, la solución se sometió a diálisis tres veces con agua destilada para eliminar el exceso de ácido fólico, el cual no reaccionó El esquema de la síntesis puede observarse en la Figura 1. Todos los reactivos (grado reactivo) se adquirieron comercialmente en Sigma-Aldrich $\AA$.

Caracterización del conjugado de grafeno y ácido fólico mediante espectroscopia Raman. Se depositó una solución de $5 \mu \mathrm{l}$ del conjugado de grafeno y ácido fólico sobre una superficie de silicio $\left(\mathrm{SiO}_{2}\right)$ y se dejó durante 15 minutos hasta su total evaporación. Posteriormente, se determinó el espectro Raman utilizando un microscopio Raman DXR ${ }^{\mathrm{TM}}$ Thermo Scientific ${ }^{\mathrm{TM}}$. El microscopio óptico se acopló a un espectrofotómetro con un ancho de banda espectral de $5 \mathrm{~cm}^{-1}$ y objetivo de 50x. La muestra se irradió con un láser de 785 nm a una potencia de $5 \mathrm{~mW}$ con un tiempo de acumulación de señales de 10 segundos. Los datos se procesaron con el programa Origin $\AA$, versión 8.1 .

Métodos computacionales. La estructura del grafeno se optimizó empleando el método semiempírico PM6 (Stewart, et al., 2007), y la del ácido fólico con la teoría de los funcionales de la densidad (DFT) (Becke, et al., 1993), y el funcional de intercambio y correlación B3LYP (Becke, et al., 1996) con la función base 6-31g(d), y se volvió a calcular con el método semiempírico PM6 (Rassolov, et al., 2001). La interacción del grafeno y el ácido fólico se llevó a cabo con el método semiempírico PM6 (Stewart, et al., 2007); todos los cálculos se hicieron con el programa Gaussian 09 (Frisch, et al., 2010).

\section{Resultados y discusión}

Caracterización del conjugado de grafeno y ácido fólico. La espectroscopia Raman (ER) se ha empleado ampliamente para el estudio de los principales modos de vibración del grafeno (Stewart, et al., 2007). La información obtenida de los espectros está básicamente relacionada con estados de agregación, de dispersión y con la funcionalización del grafeno (Dresselhaus, et al., 2010). Por ejemplo, Ni, et al. (2008) utilizaron la ER para obtener información y estudiar la formación de capas en la síntesis del grafeno. En otro estudio, Wojtoniszak, et al. (2013) utilizaron la ER para estudiar la funcionalización de grafeno con azul de metileno. La ER del conjugado de grafeno y azul de metileno registró modos de vibración a 1.314 y $1.596 \mathrm{~cm}^{-1}$ provenientes de las bandas $\mathrm{D}$ y G, lo cual representa el grado de desorden luego de modificar su estructura y el nivel de distribución de las moléculas sobre la estructura del grafeno, respectivamente. En un reporte similar, Long, et al. (2012) monitorizaron la funcionalización no covalente de grafeno con aminas mediante ER, y evidenciaron su interacción mediante el desplazamiento de la banda de vibración $G$ hacia energías menores y la disminución en la intensidad del modo de vibración $2 \mathrm{D}$.

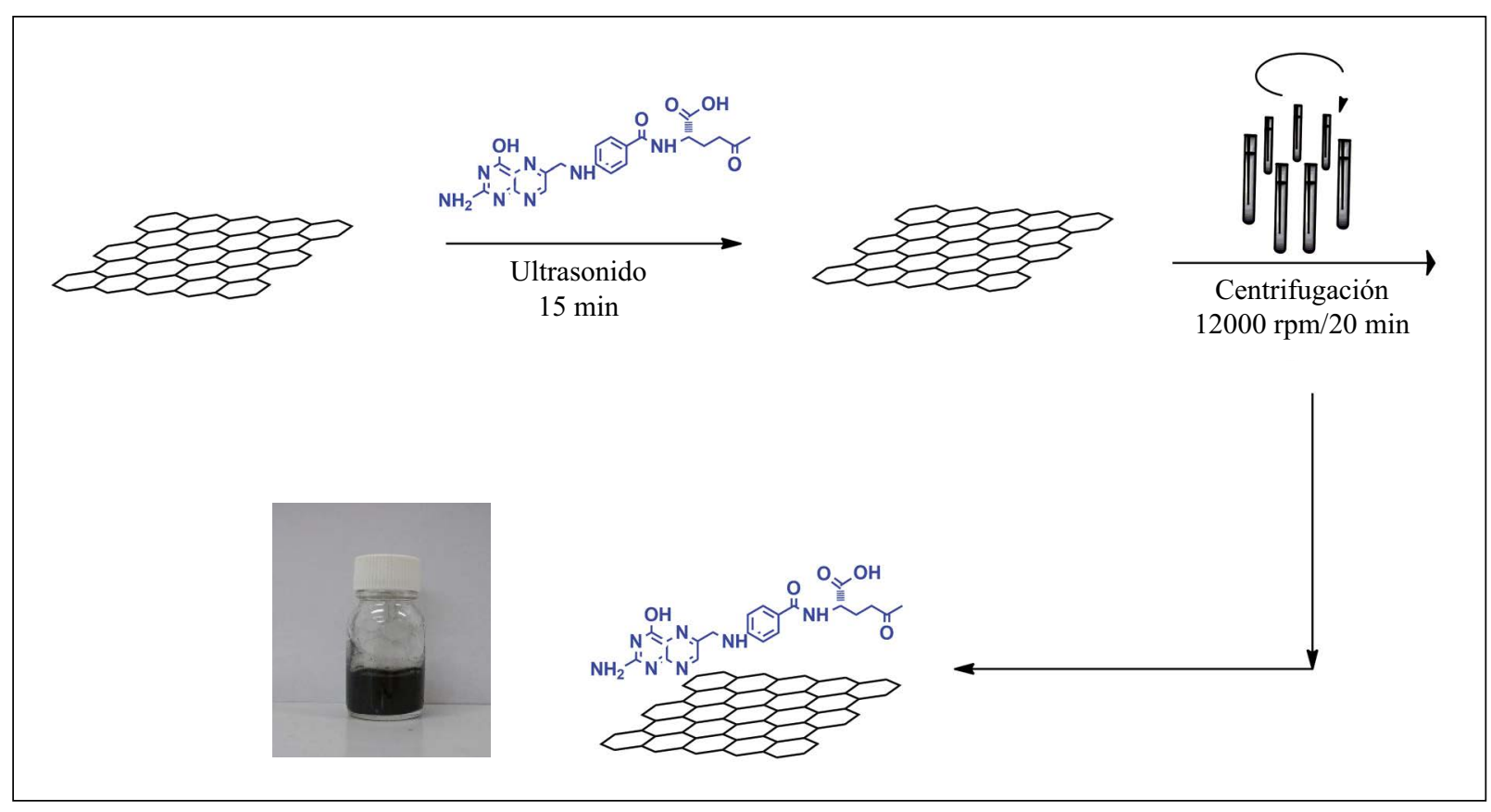

Figura 1. Esquema de la preparación del conjugado de grafeno y ácido fólico 
(A)
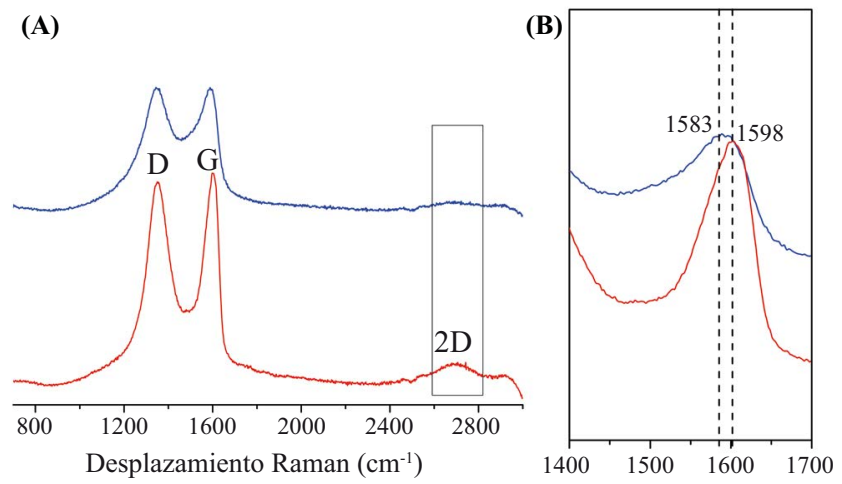

Figura 2. A. Comparación de los espectros Raman del grafeno libre (-) y del funcionalizado con ácido fólico (-). B. Detalle ampliado de la comparación del desplazamiento Raman de la banda $\mathrm{G}$ del grafeno libre (-) y el funcionalizado con ácido fólico (-).

En la Figura 2 se comparan los espectros $\mathrm{R}$ del grafeno libre y del funcionalizado con ácido fólico.

En la Figura 2A se puede apreciar claramente la presencia de dos bandas a $1.352 \mathrm{~cm}^{-1}$ (banda D) y a $1.598 \mathrm{~cm}^{-1}$ (banda G) para el caso del grafeno libre. Por otro lado, el espectro Raman del conjugado de grafeno y ácido fólico presenta las mismas bandas, pero a $1.346 \mathrm{~cm}^{-1}$ y 1.583 $\mathrm{cm}^{-1}$ para las bandas $\mathrm{D}$ y $\mathrm{G}$, respectivamente. El espectro Raman del conjugado de grafeno y ácido fólico muestra un desplazamiento de las bandas hacia valores menores en los números de onda $\left(\mathrm{cm}^{-1}\right)$, con valores de $\Delta v_{\mathrm{D}} \mathrm{y} \Delta v_{\mathrm{G}}$ de 6 y $15 \mathrm{~cm}^{-1}$, respectivamente. Probablemente la estructura del ácido fólico al interactuar con el grafeno provoca este desplazamiento en las bandas D y G, afectando de esta manera los modos de vibración del grafeno y evidenciando su funcionalización no covalente. Este mismo efecto fue observado por Li, et al. (2011) quienes funcionalizaron grafeno con ftalocianinas de galio.

Uno de los resultados más utilizados para evaluar la funcionalización de estructuras alotrópicas del carbono es el de la relación de intensidades de las bandas $\mathrm{D}$ y $\mathrm{G}, \mathrm{I}_{\mathrm{D}} /$ $\mathrm{I}_{\mathrm{G}}$ (Dresselhaus, et al., 2010). Esta relación permite monitorizar los procesos de funcionalización covalente por medio de la banda $\mathrm{D}$ resultante de la transformación de sitios $\mathrm{sp}^{2} \mathrm{a}$ $\mathrm{sp}^{3}$. En el caso de la funcionalización no covalente, la relación de intensidades $\mathrm{I}_{\mathrm{D}} / \mathrm{I}_{\mathrm{G}}$ no es muy notoria y se caracteriza por presentar valores bajos (Castillo, et al., 2013). Por otro lado, los valores de la banda $\mathrm{G}$ se utilizan para monitorizar el nivel de distribución de los sitios modificados. La funcionalización covalente de grafeno con ftalocianinas de galio (Li, et al., 2011) mostró un valor de la relación de intensidades D y $\mathrm{G}$ de 0,88 para el grafeno libre y de 1,01 para el grafeno modificado con la ftalocianina. Por otro lado, en un estudio para la modificación no covalente de nanotubos de carbono con ácido fólico apenas se registró un aumento de 0,031 en la diferencia de la relación de intensidades de los nanotubos libres y los modificados con el ácido (Castillo, et al., 2013). En la Tabla 1, se presentan los valores en las intensidades de las bandas D y G del grafeno modificado y sin modificar, además de los valores de desplazamiento Raman $\left(\mathrm{cm}^{-1}\right)$ de las bandas $\mathrm{D}$ y $\mathrm{G}$.

Como se puede apreciar, se registró un aumento en el valor de la relación de las intensidades del grafeno modificado comparada con el valor $\mathrm{I}_{\mathrm{D}} / \mathrm{I}_{\mathrm{G}}$ del grafeno sin modificar, lo cual es otra evidencia de la funcionalización del grafeno con el ácido fólico. Este aumento en el valor de $\mathrm{I}_{\mathrm{D}} / \mathrm{I}_{\mathrm{G}}$ indica un aumento en la cantidad de defectos sobre la superficie del grafeno, probablemente debido a la presencia del ácido fólico. La banda 2D es característica de las monocapas de grafeno; esta señal Raman representa un sobretono de la banda D y se utiliza para evaluar la funcionalización y el dopaje de grafeno u óxido de grafeno (Dresselhaus, et al., 2010). La disminución en la intensidad de la banda 2D del conjugado de grafeno y ácido fólico es un indicio del aumento de defectos superficiales del grafeno, lo cual está relacionado, a su vez, con la funcionalización con ácido fólico. Estudios similares muestran, por ejemplo, que la disminución en la intensidad de la banda 2D está relacionada con la funcionalización de grafeno con ácido fólico y óxido de cinc (Ni, et al., 2008).

Tabla 1. Valores de la relación de intensidades de las bandas D y G para el grafeno libre y el modificado con ácido fólico

\begin{tabular}{|c|c|c|c|}
\hline \multirow[t]{2}{*}{ Muestra } & \multicolumn{2}{|c|}{$\begin{array}{l}\text { Desplazamiento Raman } \\
\qquad\left(\mathrm{cm}^{-1}\right)\end{array}$} & \multirow[t]{2}{*}{$\mathbf{I}_{\mathbf{D}} / \mathbf{I}_{G}$} \\
\hline & Banda D & Banda $\mathbf{G}$ & \\
\hline Grafeno & $1352 \pm 5,2$ & $1598 \pm 3,2$ & 0,84 \\
\hline Grafeno y ácido fólico & $1326 \pm 2,8$ & $1583 \pm 4,5$ & 0,87 \\
\hline
\end{tabular}

Triplicados; nivel de confianza del $95 \%$

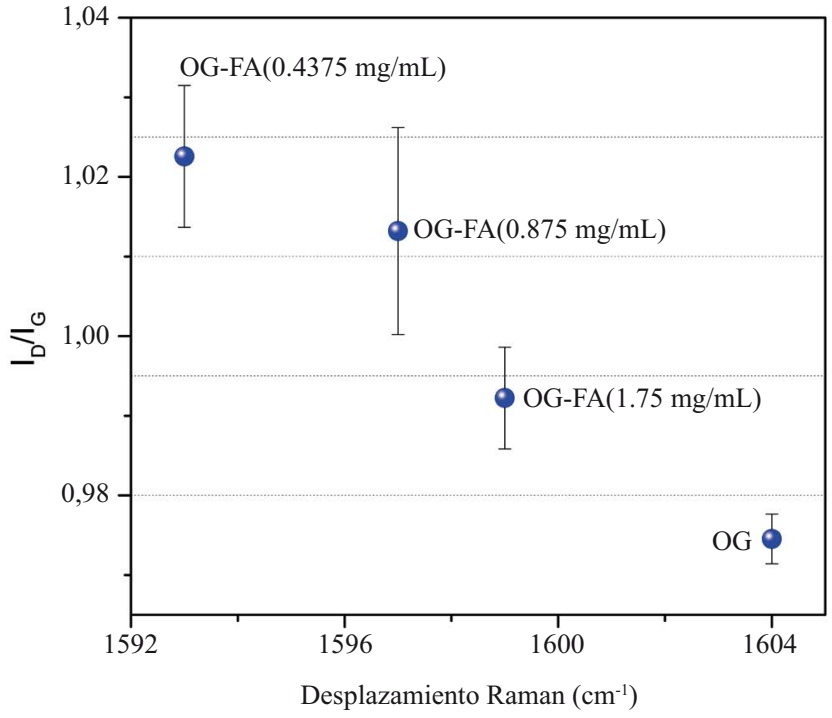

Figura 3. Relación de intensidades de las bandas D y G en función de la banda $\mathrm{G}$ para diferentes concentraciones de óxido de grafeno 
En la Figura 3 se presenta la relación de intensidades $I_{D} /$ $I_{G}$ en función de la banda $G$ para diferentes concentraciones de grafeno libre y del funcionalizado con ácido fólico a una concentración constante de $6,5 \mathrm{mM}(\mathrm{n}=10)$. Como se aprecia en la figura, a medida que disminuye la cantidad de grafeno el número de defectos aumenta debido a que el ácido ocupa un mayor número de posiciones en la estructura del grafeno. Por otro lado, se aprecia nuevamente el desplazamiento hacia números de ondas menores a medida que el ácido fólico interactúa con el grafeno.

Con el propósito de simular la interacción entre el ácido fólico y el grafeno la estructura electrónica del ácido fue optimizada. como se observa en la Figura 4

En la estructura optimizada del ácido fólico se ven el ácido glutámico y la parte p-aminobenzoico (paba) en el mismo plano molecular, además, el ácido glutámico posee un ángulo diedro de $175,8^{\circ}(\mathrm{C} 13-\mathrm{C} 10-\mathrm{C} 7-\mathrm{C} 6)$ y otro ángulo diedro formado por la unión entre este y la parte paba, con un valor de $187,7^{\circ}$ (C6-N17-C19-C20), en tanto que el ácido glutámico $\alpha$ está fuera del plano, con un diedro de 282,4 ${ }^{\circ}$ (C4-C6-N17-C19). Estos resultados concuerdan con los reportados anteriormente por Castillo, et al. (2013).

Los métodos computacionales híbridos se han empleado como complemento de los resultados experimentales para simular diversos tipos de interacciones entre formas alotrópicas de carbono (grafeno, nanotubos de carbono, etc.) y diferentes tipos de biomoléculas. En la Tabla 2 Se pueden apreciar estudios recientes de la aplicación de métodos computacionales para simular interacciones del grafeno y varios tipos de moléculas.

En este estudio y por medio del método semiempírico PM6 se trabajó con una lámina de grafeno de una longitud 20x20 Å de tal forma que el ácido fólico tuviese movilidad en la lámina de grafeno y no interaccionara con carbonos de la orilla que tienen hidrógenos. En el modelo A el AF se ubicó en el centro de la lámina de grafeno, mientras en el modelo B en la orilla (ver figura 5).

El modelo teórico basado en el método computacional PM6 corrobora que la formación del complejo grafeno-AF ocurre por medio de interacciones débiles tipo puente de hidrogeno (Jefrey, 1997; Martz, 2004). Las interacciones de tipo puentes de hidrógeno se presentan cuando un átomo donador se enlaza covalentemente a otro átomo aceptor electronegativo. De acuerdo con lo anterior las distancias atómicas donor-aceptor en el rango 2.2-2.5 $\AA$ son consideradas como interacciones covalentes. Por otro lado, en el rango 2.5-3.2 $\AA$ son moderadamente covalentes y en el rango 3.2-4.0 $\AA$ se consideran como interacciones débiles electrostáticas (Jefrey, 1997). Según lo anterior y como se aprecia en las distancias calculadas entre el ácido fólico y la superficie del grafeno la interacción ácido fólico -grafeno es una interacción no covalente (Figura 6).

El modelo teórico basado en el método computacional PM6 corrobora que la formación del complejo de grafeno y

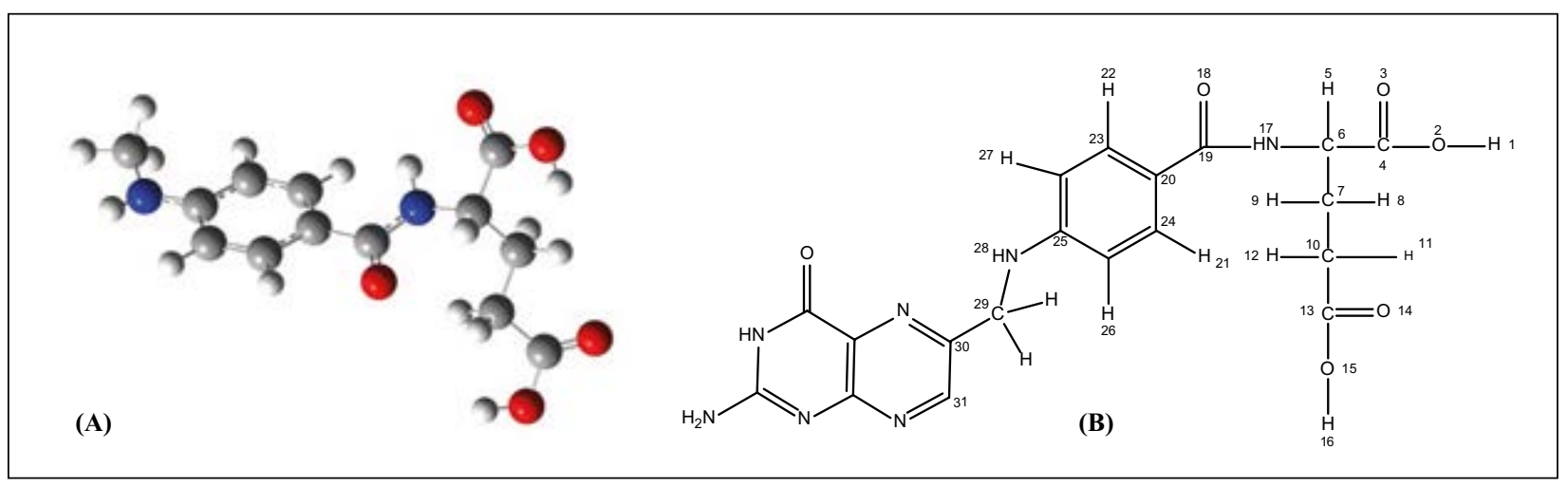

Figura 4. A. Geometría optimizada de la estructura del ácido fólico. B. Estructura química del ácido fólico

Tabla 2. Comparación de métodos computacionales para el estudio de la funcionalización de grafeno con diferentes moléculas

\begin{tabular}{lll}
\hline Método & Interacción & Referencia \\
\hline DFT ab initio & Grafeno-areno & (Zhao, et al., 2012) \\
DFT & Grafeno-boro & (Manna, et al., 2013) \\
GGA-DFT & Grafeno-ADN & (Xu, et al., 2014) \\
MP2 & Grafeno-naftaleno-ADN & (Cho, et al., 2013) \\
LDA-DFT & Grafeno-bisulfato de sodio & (Ayala, et al., 2012) \\
\hline
\end{tabular}

GGA: aproximación del gradiente generalizado; LDA: aproximación de la densidad local MP2: método Moller-Plesset post Hartree-fock. 
(A)

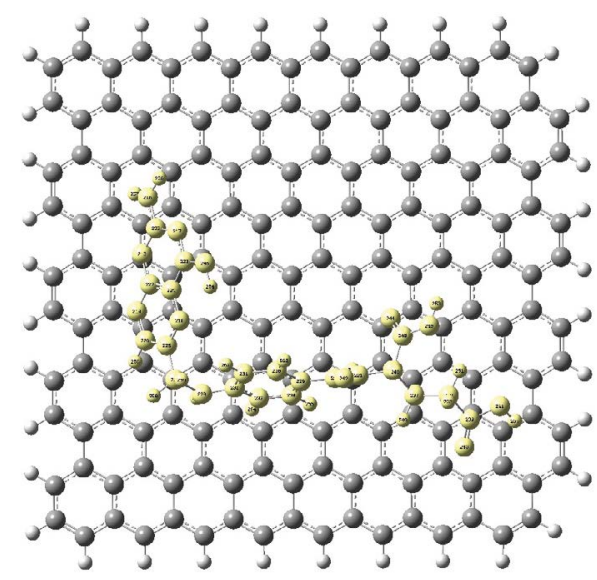

(B)

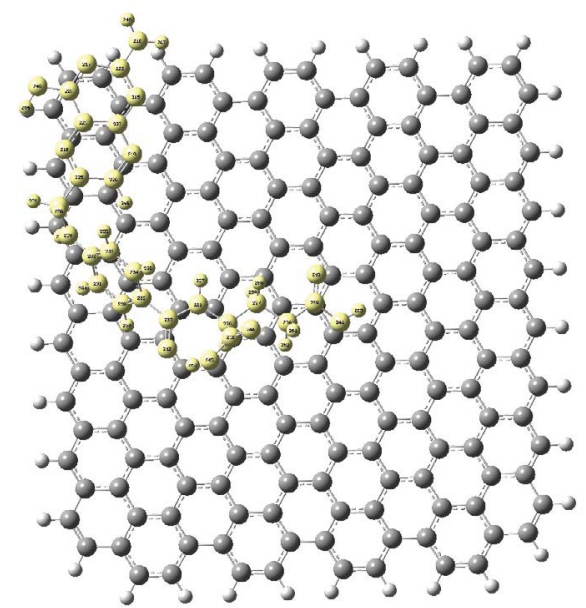

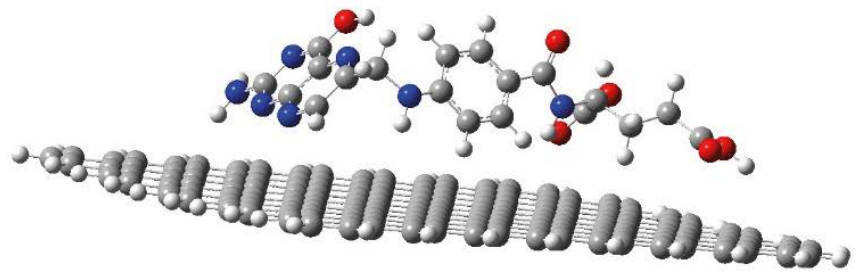
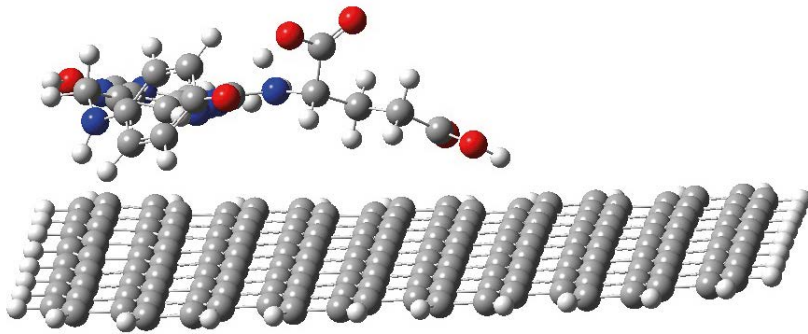

Figura 5. Interacciones del grafeno y el ácido fólico. Modelo A. Ácido fólico en el centro de la lámina de grafeno. Modelo B. Ácido fólico en la orilla

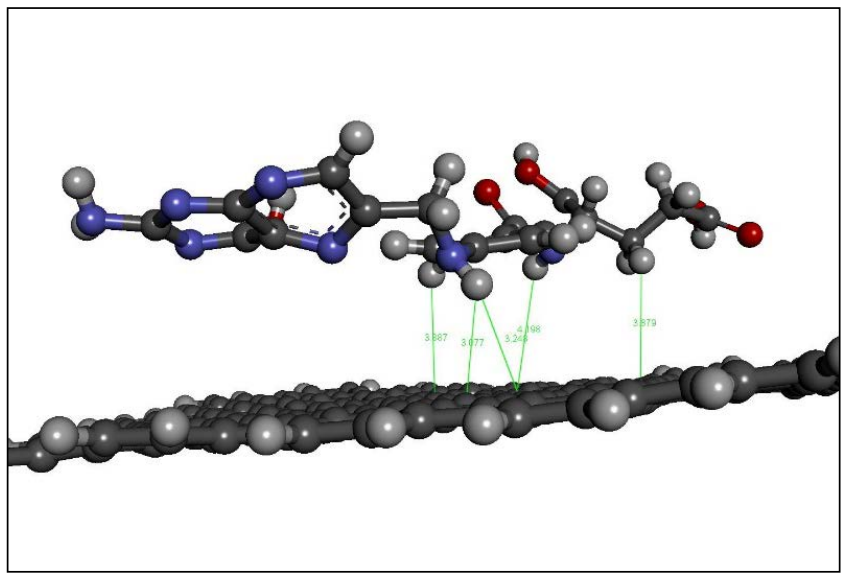

Figura 6. Distancias interatómicas representativas de la interacción del ácido fólico y el grafeno

ácido fólico ocurre por medio de interacciones débiles del tipo de puente de hidrogeno (Jefrey, 1997; Martz, 2004). Este tipo de interacciones se presentan cuando un átomo donador se enlaza de forma covalente con otro átomo aceptor electronegativo, de manera que las distancias atómicas entre donador y aceptor en el rango de 2,2 a $2,5 \AA$ se consideran como interacciones covalentes. Por otro lado, en el rango de 2,5 a $3,2 \AA$ son moderadamente covalentes y en el rango de 3,2 a $4,0 \AA$ se consideran como interacciones débiles electrostáticas (Jefrey, 1997). Según lo anterior, y como se aprecia en las distancias calculadas entre el ácido fólico y la superficie del grafeno, la interacción de los dos es no covalente.

Como se observa en la Figura 6 existen dos modelos: el modelo A se caracteriza por la interacción de los átomos de hidrógeno de los anillos pteridínico y paba del ácido fólico con los electrones de los anillos aromáticos del grafeno. Esta interacción posee un valor de energía de $473.372 \mathrm{Kcal}$ $\mathrm{mol}^{-1}$. El modelo B se caracteriza por la interacción de los electrones $\pi$ de la pteridina y del anillo aromático del paba con la nube electrónica del grafeno, por lo tanto es una interacción no covalente del tipo de apilamiento $(\pi-\pi)$. Esta interacción presentó un valor de energía de $474.454 \mathrm{Kcal}$ $\mathrm{mol}^{-1}$. Si se comparan los valores de energía de los modelos se concluye que el modelo A presentó el valor de energía más bajo y, por lo tanto, fue el más estable, y se caracterizó 
por la interacción de los hidrógenos del ácido fólico con la nube electrónica del grafeno, que, al estar en la orilla, no se favorecen energéticamente

\section{Conclusiones}

El conjugado de grafeno y ácido fólico se formó con interacciones de tipo no covalente, lo cual se demostró empleando espectroscopia Raman mediante los valores de la relación $\mathrm{I}_{\mathrm{D}} / \mathrm{I}_{\mathrm{G}}$ y se sustentó con cálculos teóricos que evidenciaron interacciones del tipo de puente de hidrógeno. El conjugado obtenido y caracterizado de esta manera podría utilizarse para la detección de células malignas con sobreexpresión de receptores de folato, o en la elaboración de sistemas de detección rápida de células cancerígenas.

\section{Agradecimientos}

Los autores expresan su agradecimiento al Department of Micro and Nanotechnology, Technical University of Denmark, y a The Danish Concil for Independent Research's NAnoPLASmonic Sensors (NAPLAS) Sapere Aude Project, por la financiación de este estudio.

\section{Contribución de los autores}

Angie Orduz, ejecutó y llevo a cabo la mayor parte de los experimentos relacionados con la síntesis y caracterización del conjugado grafeno-ácido fólico. Ciro E. Rozo, ejecutó toda la parte relacionada con los métodos computacionales para la caracterización del grafeno-ácido fólico. John J. Castillo, coordinó todas las actividades y escribió el artículo.

\section{Conflicto de intereses}

Los autores declaran que no tienen conflictos de intereses.

\section{Referencias}

Allen, M., Tung, M., Kaner, R. (2010). Honeycomb carbon: A review of graphene. Chem Rev. 110: 132-145.

Ayala, I.G., Cordero, N.A. (2012) Interaction of sodium bisulfate with mono- and bi-Layer graphene. J Nanoparticle Res. 14: 1-7.

Balapanuru, J., Yang, J.X., Xiao, S., Bao, Q., Jahan, M., Polavarapu, L., Wei, J., Xu, Q.H., Loh, K.P. (2010). A graphene oxide-organic dye ionic complex with DNAsensing and optical-limiting properties. Angew Chemie Int Ed. 49: 6549-53.

Becke, A.D. (1993). Density-functional thermochemistry. III. The role of exact exchange. J Chem Phys. 98: 5648.

Becke, A.D. (1996). Density-functional thermochemistry. IV. A new dynamic correlation functional and implications for exact-exchange mixing. J Chem Phys. 104: 1040-6.

Bose, S., Kuila, T., Mishra, A.K., Kim, N.H., Lee, J.H. (2011) Preparation of non-covalently functionalized graphene using 9-anthracene carboxylic acid. Nanotechnology. 22: 405603.

Casiraghi, C. (2011). Raman intensity of graphene. Phys Status Solidi Basic Res. 248: 2593-7.

Castillo JJ, Svendsen WE, Rozlosnik N, Escobar P, Martínez F. Castillo-León J. (2013). Detection of cancer cells using a graphene modified peptide nanotube-folic acid. Analyst. 138: $965-972$.
Castillo J.J., Rindzevicius T., Novoa L. V., Martínez F., Svendsen W.E., Rozlosnik N., Boisen A., Escobar, P., Martínez, F., Castillo, J. (2013). Non-covalent conjugates of singlewalled carbon nanotubes and folic acid for interaction with cells over-expressing folate receptors. J Mater Chem B. 1: 1475-81.

Castillo, J.J., Rindzevicius, T., Wu, K., Rozo, C.E., Schmidt, M.S., Boisen, A. (2015). Silver-capped silicon nanopillar platforms for adsorption studies of folic acid using surface enhanced Raman spectroscopy and density functional theory. J Raman Spect. 46: 1087-1094.

Castillo, J.J., Rozo, C.E., Castillo-León, J., Martínez, F., Rindzevicius, T., Svendsen, W.E., Rozlosnik, N. (2013). Computational and experimental studies of the interaction between single-walled carbon nanotubes and folic acid. Chem Phys Lett. 564: 60-4.

Castillo, J.J., Torres, M.H., Molina, D.R., Castillo-León, J., Martínez, F., Escobar P., Svendsen, W.E. (2012) Monitoring the functionalization of single-walled carbon nanotubes with chitosan and folic acid by two-dimensional diffusion-ordered NMR spectroscopy. Carbon. 50: 2691-7.

Cho, Y., Min, S.K., Yun, J., Kim, W.Y., Tkatchenko, A., Kim, K.S. (2013). Noncovalent interactions of DNA bases with naphthalene and graphene. J Chem Theory Comput. 9: 2090-6.

Choi, E.Y., Han, T.H., Hong, J., Kim, J.E., Lee, S.H., Kim, H.W., Kim, S.O. (2010). Noncovalent functionalization of graphene with end-functional polymers. J Mater Chem. 20: 1907 .

Dresselhaus, M.S., Jorio, A., Hofmann, M., Dresselhaus, G., Saito, R. (2010). Perspectives on carbon nanotubes and graphene Raman spectroscopy. Nano Lett. 10: 751-8.

Frisch, M., Trucks, G., Schlegel, H., Scuseria, G., Robb, J., Cheeseman, J. (2010). Gaussian 09, Revision B.01, Inc. Wallingford, CT, 2010.

Hu, C., Liu, Y., Qin, J., Nie, G., Lei, B., Xiao, Y. (2013). Fabrication of reduced graphene oxide and sliver nanoparticle hybrids for Raman detection of absorbed folic acid: A potential cancer diagnostic probe. ACS Appl Mater Interfaces. 12: 4760-4768.

Jeffrey, A. (1997). An Introduction to Hydrogen Bonding. Oxford University Press. p. 56-71.

Martz, E. (2004). Help, Index \& Glossary for Protein Explorer. http://www.umass.edu/microbio/chime/pe_beta/pe/ protexpl/igloss.htm?q=microbio/chime/explorer/igloss. htm

Kamińska, A., Dzięcielewski, I., Weyher, J.L., Waluk, J., Gawinkowski, S., Sashuk, V., Fiałkowski, M., Sawicka, M., Suski, T., Porowski, S., Hołyst, R. (2011). Highly reproducible, stable and multiple regenerated surfaceenhanced Raman scattering substrate for biomedical applications. J Mater Chem. 21: 8662.

Li, Y.X., Zhu, J., Chen, Y., Zhang, J., Wang, J., Zhang, B., He, J., Blau, W.J. (2011). Synthesis and strong optical limiting response of graphite oxide covalently functionalized with gallium phthalocyanine. Nanotechnology. 22: 205704.

Long, B., Manning, M., Burke, M., Szafranek, B.N., Visimberga, G., Thompson, D., et al. (2012). Non-covalent functionalization of graphene using self-assembly of alkane-amines. Adv Funct Mater. 22: 717-25. 
Manna, A.K., Pati, S.K. (2013). Computational studies on noncovalent interactions of carbon and boron fullerenes with graphene. ChemPhysChem. 14: 1844-52.

Ni, Z., Wang, Y., Yu, T., Shen, Z. (2008). Raman spectroscopy and imaging of graphene. Nano Res. 1: 273-91.

Rassolov, V.A. (2001). 6-31G* basis set for third-row atoms. J Comput Chem. 22: 976-84.

Stewart, J.J. (2007). Optimization of parameters for semiempirical methods V: Modification of NDDO approximations and application to 70 elements. J Mol Model. 13: 1173-213.
Wojtoniszak, M., Rogińska, D., Machaliński, B., Drozdzik, M., Mijowska, E. (2013). Graphene oxide functionalized with methylene blue and its performance in singlet oxygen generation. Mater Res Bull. 48: 2636-9.

Xu, Z., Meher, B.R., Eustache, D., Wang, Y. (2014). Insight into the interaction between DNA bases and defective graphenes: Covalent or non-covalent. J Mol Graph Model. 47: 8-17.

Zhao, J.X., Wang, H.X., Gao, B., Wang, X.G., Cai, Q.H., Wang, X.Z. (2012). Chemical functionalization of graphene via aryne cycloaddition: A theoretical study. J Mol Model. 18: 2861-8. 